\title{
Breve reflexión sobre la razonabilidad de la Información Financiera y la Tributación
}

\author{
Bastidas Canelones, Jorge \\ *Universidad de los Andes, Venezuela \\ E-mail: jorgebastidas1983@gmail.com \\ https://orcid.org/0000-0002-5076-0249
}

Recibido: 17 de diciembre de 2020

Aprobado: 22 de diciembre de 2020

\section{Resumen}

En esta reflexión se tratará de evidenciar primeramente el carácter interdisciplinario entre Contabilidad Financiera y Tributación, como preámbulo a develar las tensiones que se producen en la relación diaria y práctica entre estas dos disciplinas. Se comentará en una reflexión autocrítica sobre la im-posibilidad del modelo de regulación contable internacional de conseguir que la información que se produce a partir de la aplicación del modelo sea razonable y las consecuencias que tiene para la Tributación tener que usar esa información para efectos de determinación de obligaciones materiales, ya que, para criterio del investigador, esa información no está ajustada a la razón por no representar fielmente los hechos y transacciones de la empírea económica; lo cual hemos denominado como tensión otológica. Probablemente la tensión que produce la información razonable construida a partir del uso del modelo contable internacional en su interacción con la Tributación en las distintas jurisdicciones donde se relacionen, se produzca por lo disímil de su sistema de creencias. Para esta investigación no se pueden develar todas las tensiones por razones de espacio, pero, en criterio del investigador, la tensión llamada Información Razonable Vs. Tributación es un elemento probatorio para afirmar que ambos sistemas conviven en conflictos y que no basta subsanar las diferencias a través de las conciliaciones tributarias.

Palabras clave: Contabilidad Financiera, Razonabilidad, Tributación, Interdisciplina. 


\title{
Brief reflection on the reasonableness of Financial Information and Taxation
}

\begin{abstract}
This reflection we will try to first demonstrate the interdisciplinary nature between Financial Accounting and Taxation, as a preamble to uncovering the tensions that occur in the daily and practical relationship between these two disciplines. It will be commented in a self-critical reflection on the impossibility of the international accounting regulation model to ensure that the information produced from the application of the model is reasonable and the consequences for Taxation of having to use that information for tax purposes. determination of material obligations, since, in the opinion of the researcher, this information is not adjusted to the reason for not faithfully representing the facts and transactions of the economic empyrean; which we have termed as otological tension. Probably the tension produced by the reasonable information built from the use of the international accounting model in its interaction with Taxation in the different jurisdictions where they are related, is produced by the dissimilarity of their belief system. For this investigation, not all tensions can be revealed for reasons of space, but, in the researcher's opinion, the tension called Reasonable Information Vs. Taxation is an element of evidence to affirm that both systems coexist in conflicts and that it is not enough to correct the differences at through tax conciliations.
\end{abstract}

Keywords: Financial Accounting, Reasonable, Taxation, Interdiscipline.

\section{Introducción}

\subsection{La interacción disciplinal}

Antes de comentar sobre la razonabilidad de la Información Financiera (que plantea la Contabilidad Financiera) y la Tributación es preciso recordar que la Contabilidad Financiera y la Tributación son disciplinas que interactúan constantemente. Estas disciplinas están íntimamente relacionadas, de hecho, la contabilidad es interdisciplinaria desde sus inicios (Lowe y Tucker 1976) y, quizás, en la relación con el tributo en la praxis disciplinal, se hace más evidente la interdisciplinariedad pues reconocen y representan conjuntamente hechos presentes en la empírea económica. Por ello, sostengo que la Contabilidad Financiera y la Tributación, se han visto obligadas a interactuar entre sí y con otras ciencias sociales a pesar de que los esquemas de poder que producen los discursos contables y tributarios procuran, a mi entender, soslayar la existencia de una y la otra en la producción del conocimiento desde sus distintos linderos científicos.

La Tributación necesita de las manifestaciones de riqueza que cuantifica metodológica y científicamente la Contabilidad Financiera para dar magnitud numérica a la materia gravable $\mathrm{y}$, en ocasiones, a la obligación tributaria material. Por su parte, la Contabilidad Financiera solo necesita de la Tributación para reconocer el monto de las acreencias tributarias con el sujeto pasivo en los Estados Financieros. Si en un supuesto negado, la Tributación desaparece como disciplina, la Contabilidad Financiera puede seguir funcionando e interactuando con otras ciencias sociales, no obstante, si la Contabilidad Financiera desapareciera, la Tributación se vería obligada desarrollar criterios propios para reconocer, medir y presentar los hechos económicos y transacciones que se dan en el entorno empresarial.

En esta interacción disciplinal hay conceptos tributarios como el Hecho Imponible, la Base Imponible, la Materia Gravable, entre otros, con los cuales la Contabilidad Financiera se relaciona de forma directa. Si bien es cierto que la materia gravable es una realidad independiente del mundo jurídico y como entidad, antecede al hecho imponible y a la base imponible, no es menos cierto que para que exista como hecho económico, debe tener un correlato monetario subyacente. 
La materia gravable puede tener "una regulación específica en el mundo jurídico como objeto de contratos o como objeto imponible pero no es una creación del derecho. Incluso, su denominación puede ser creación del derecho" (Hevia 2010 p. 42), de la norma contable o del lenguaje común; sin embargo, existe como materia, como entidad, como un "hecho bruto" (Searle 1995), sin que se le incluya como objeto del gravamen regulado por una norma jurídica con contenido tributario, pero al incluírseles, necesita de una disciplina que le asigne valor para que tenga representatividad en la empírea económica.

Una maquinaria, una herramienta, un camión, una casa, un terreno, el efectivo, el vehículo, la materia prima, los insumos, un software, etc., como entidades, no son creaciones del derecho ni de la contabilidad, son materias gravables, pero a su vez son entidades físicas (aunque el lenguaje, como construcción social, es la que determina su significante) Son creaciones materiales del hombre, incluso, pudieran ser realidades pertenecientes a un campo normativo distinto al jurídico o al contable, empero, todas tienen un código lingüístico común, son expresadas y reconocidas en términos monetarios y la Contabilidad Financiera coadyuva en la asignación del valor a las materias gravables, es decir, es la interfaz entre su representación física pura y su representación financiera, pues la reflejará con un correlato monetario determinado.

Señala Hevia (2013) que la existencia de "la materia gravable no depende de la juridificación y existe independientemente de la delimitación del presupuesto imponible y de su cuantía" (ob. Cit.), sin embargo, sin un valor asociado será inútil para el entorno comercial y tributario. Una persona natural o jurídica que sea propietaria de un edificio que no tenga un correlato monetario asignado, no tendrá sino una estructura de concreto sin utilidad en el campo de los negocios, pues no se contarían con valores que faciliten el proceso del goce, usufructo o intercambio del bien en la realidad comercial.

En un sentido práctico, la Tributación determina cuál materia será la gravable, cuál es el supuesto de imposición y cómo es su proceso de medición, es decir, éstas últimas variables estarán en función de la materia gravable, pues si no hay materia que gravar "no habrá hecho imponible, ni base imponible. Así por ejemplo, sin un inmueble, no hay objeto para vender, ni tampoco habrá un bien para determinar un enriquecimiento por la diferencia entre su precio de venta y su costo fiscal" (Ob. Cit.) y muchos menos una hipotética obligación tributaria.

Un ejemplo para visualizar la relación Tributación - Contabilidad Financiera en la que se incorporan los conceptos descritos es en la determinación del Impuesto sobre la Renta (ISR en adelante) La verificación de la existencia de la Renta Neta en el ISR es el Hecho Imponible, la cuantificación de la Renta Neta a partir de la disposiciones de la ley y de los valores y partidas contables admisibles sería la Base Imponible y, el inmueble, el activo fijo, el objeto, el derecho o el bien mueble, sobre el cual recae el hecho imponible, es la materia gravable.

En algunas jurisdicciones, por ejemplo, se parte del valor según libros (Base Financiera) para determinar si hubo una ganancia o pérdida fiscal por la hipotética venta de un inmueble, la cual generaría, dependiendo del país donde ocurra, un impuesto a la renta. Para este caso, salvo disposición en contrario, se necesitaría de la Contabilidad Financiera para determinar si se configuró el presupuesto de hecho que de origen a la obligación tributaria, es decir la obtención de una renta, el cual surge por la diferencia positiva entre el ingreso bruto producto de la venta de ese activo y su costo, lo cual, según lo descrito, es el Hecho Imponible.

También la requeriría para determinar el monto a gravar (Base Imponible) a partir de la inclusión o exclusión de valores reconocidos en contabilidad de ingresos y costos y, a su vez, necesitará a la Contabilidad para valorar inicialmente "el activo, objeto, derecho, bien mueble o inmueble que interviene en la verificación del hecho imponible, lo cual es la Materia Gravable" (Ob. Cit.); sin esta valoración de la materia gravable sería imposible tener un correlato monetario que permita determinar su costo fiscal. Esto da muestra de la dimensión interdisciplinaria en que actúan estas dos disciplinas.

La interacción disciplinal también puede 
observarse para el caso de la transmisión por causa de muerte del titular de un bien. En Venezuela, para la determinación del Impuesto sobre Sucesiones, no se establecen definiciones específicas para Activos, Pasivos o Patrimonio, simplemente se asume en la ley que quien la aplique conoce el concepto de Activo (tal vez el legislador tiene la creencia de que en el Derecho el concepto de Activo debe entenderse de la misma forma que se hace en Contabilidad) Este impuesto se calcula al restar al Activo Sucesoral el Pasivo Sucesoral, obteniendo así el Patrimonio Neto Hereditario (Art. 15 al 26, y 59 Ley de ISSDYDRC)

En los ejemplos anteriores se visualiza como en este proceso de categorización y de medición la Tributación debe apoyarse de la Contabilidad Financiera. Es así como se necesita de la Contabilidad Financiera para conocer en un momento inicial o a la fecha del periodo que se reporta, el valor de una determinada materia gravable, pues la existencia de un bien o derecho tiene implícito un componente cuantitativo que la Contabilidad se encarga de representar (relación existencia -valoración) Si no hay materia gravable que cuantificar (la cuantifica la Contabilidad), no habrá objeto para vender que origine un ingreso (lo cuantifica la Contabilidad) y luego una renta (Hecho Imponible), por tanto, tampoco habrá un objeto que sea susceptible de imposición y de cuantificación de esa imposición (Base Imponible)

Para que la Tributación obvie a la Contabilidad Financiera en este proceso de categorización debe desarrollar sus propias técnicas de medición, criterios de reconocimiento de transacciones, reglas de representación de la realidad económica, etc. Mientras tanto, la Contabilidad Financiera estará presente en estas tres dimensiones. Cabe aclarar que, por ahora, la Tributación no tiene como objetivo disciplinal el desarrollo de teorías, conceptos y métodos propios para el reconocimiento y medición de hechos económicos o transacciones que les permita representarlos en Activos, Pasivos, Ingresos, Costos o Gastos y que tengan solo consecuencias tributarias.

En ocasiones la Tributación, basándose en el principio de reserva legal establecido en el corpus normativo de una determinada jurisdicción, decide la forma y el grado en que debe apoyarse o no en otros conocimientos, como, por ejemplo, en el conocimiento contable. Aquí vemos la justificación del por qué existen Bases Financieras y Bases Contables para la medición de una determinada partida que sea de interés para la Tributación.

La descripción hecha hasta ahora sugiere que la relaciónentrelaContabilidadFinancierayTributación es estrecha. Si bien es cierto, la Tributación tiene como finalidad contribuir al gasto del Estado pues representa la forma de obtener dichos ingresos para el cumplimiento de los fines sociales del Estado, es decir, tiene por objeto recaudar los fondos que el Estado necesita para su funcionamiento y se vale de las leyes para este fin, en ese proceso de diseñar la forma de obtener los ingresos para una determinada jurisdicción, la Tributación debe inexorablemente apoyarse en otras disciplinas para darle forma a los procedimientos de exacción y para configurar la relación jurídico-tributaria en un Estado de Derecho (Sociología, Derecho, Ciencias Políticas, Economía, Contabilidad Financiera, Psicología Social, entre otras), por ello, cabe afirmar que la Tributación no solo es interdisciplinaria con la Contabilidad Financiera, también es multidimensional.

Por su parte, la Contabilidad Financiera, aunque en menor grado, necesita de la Tributación para el cumplimiento de sus fines pues es quien le suministra la información sobre los Pasivos Tributarios que deben ser reconocidos en el Balance. Es decir, los Pasivos Tributarios Corrientes o No Corrientes surgen a partir de una imposición legal existente en el marco de un sistema tributario (nullum tributum sine lege) y no propiamente por el intercambio comercial que realice la entidad con sus clientes, acreedores, entre otros. No obstante, es de reconocer que si la Tributación deja de existir, la Contabilidad Financiera (o la Contabilidad) seguirían existiendo como disciplina.

\subsection{La interacción disciplinal en la región}

Dando una mirada a otros países de la región también se puede notar la interacción en la praxis disciplinal de ambos conocimientos. Para el caso 
peruano, el art. 33 del Reglamento de la Ley de Impuesto a las Rentas establece que

La contabilización de operaciones bajo principios de contabilidad generalmente aceptados, puede determinar, por la aplicación de las normas contenidas en la Ley, diferencias temporales y permanentes en la determinación de la renta neta. En consecuencia, salvo que la Ley o el Reglamento condicionen la deducción al registro contable, la forma de contabilización de las operaciones no originará la pérdida de una deducción.

Señala Durán y Mejías (2016) que producto de la aplicación del art. 33 del Reglamento de la ley del ISR citada se establecen las siguientes reglas:

a) La determinación del ISR se hace sobre la base de lo que señalen la LIR y su Reglamento.

b) La contabilización de las operaciones de las personas jurídicas, es decir, la elaboración de los EE. FF., se hace - de conformidad con el art. 223 de la Ley General de Sociedades (LGS) - bajo principios de contabilidad generalmente aceptados (que el Consejo Normativo de Contabilidad ha interpretado que son las NIIF vigentes en el Perú)

c) La determinación del ISR por parte del contribuyente se hace en la declaración anual del ISR, partiendo del resultado contable (para tal efecto deben consignarse en la declaración los EE. FF. del ejercicio), al que deben practicarse (consignarse en la declaración jurada) ajustes correctivos (denominados comúnmente como "agregados y deducciones" o "reparos aditivos y reparos deductivos") con base en la aplicación de la regla a) anterior.

De estas reglas que proponen Durán y Mejías (2016) se puede concluir que para ellos el resultado que se plasma en los reportes contables debe ser el punto de partida de la determinación del ISR en el Perú, y que funciona "como una "base fáctica", un "medio de prueba" o una "base metodológica", pero no como norma jurídica con el fin de determinar la base imponible del impuesto" (p. 6)

Sobre la postura de Durán y Mejías (2016) es necesario aclarar que el hecho de que la Tributación necesite de la Contabilidad Financiera no significa que las empresas deban adaptar sus registros contables a los requerimientos fiscales, pues la empresa debe utilizar un Marco de Referencia de Información Financiera aplicable a la jurisdicción en donde tenga su establecimiento permanente, lo que tampoco significa que de cara al futuro, no se construyan epistemes interdisciplinales que hagan que los procesos de comunicación sean más efectivos, ya que como se describió, en ocasiones la información provenientes de una u otra disciplina es procesada y presentada de forma distinta.

No existe separación absoluta suponiendo independencia disciplinal entre la Contabilidad Financiera y la Tributación. Así lo considera Romero-Muci (2011) al reconocer que:

Una separación absoluta entre ambos modelos contables (financiero y fiscal) no se da en la realidad de los sistemas legales, pues de alguna manera siempre se presenta una interrelación entre ambos sistemas de fuentes. La razón es eminentemente práctica: no se pueden llevar varias contabilidades a la vez. Hay una sola contabilidad y las demás son complementarias de la principal, pues sólo puede haber una situación financiera. Lo contrario atentaría contra la confiabilidad y comparabilidad de la información

Y, a pesar de que los practicantes pudieran considerar como propios conceptos tradicionalmente de otras disciplinas, eso no hace que desaparezca su interrelación. Así, la Corte Suprema de la Argentina ha expresado que el concepto del devengo es propio del Derecho. Esto consta en el caso "Compañía Tucumana de Refrescos S.A." en el que se aclara el alcance de este concepto tipificado en la Ley de Impuesto a las Ganancias de ese país, a los efectos de su aplicación en un determinado año fiscal. A tenor de lo dicho, para lo corte 
'Devengar' es un concepto general del derecho empleado usualmente para dar cuenta de la circunstancia misma del nacimiento $u$ origen de un derecho de contenido patrimonial. Alude, en tal sentido, al fenómeno mismo de la génesis de un derecho. En dicha inteligencia, cuando el art. 18 de la Ley de Impuesto a la Ganancia emplea el citado término lo hace para que se realice la imputación de las ganancias y los gastos al ejercicio en que acaecieron los hechos jurídicos que son su causa, con independencia de otras consideraciones que no surjan de lo dispuesto por la ley (sentencia leída en digital, Buenos Aires, 24-5-11)

Esta postura de la Corte Suprema de la Argentina, si bien se extralimita en la interpretación del concepto del 'devengo' al apropiárselo de la contabilidad, demuestra la necesidad de mejorar los procesos de comunicación interdisciplinales y hacerlos más efectivos, en vista de que es innegable que en la praxis existen zonas de dependencia e independencia entre ambos conocimientos.

En este orden, Ferullo y otros (2006) destacan la existencia de un grado de compatibilidad necesario entre el discurso tributario y el contable que permita a ambas áreas del conocimiento el cumplimiento de sus fines y así garantizar una comunicación efectiva entre ambas disciplinas. Con base en la legislación colombiana, identifica los nichos de interacción de ambas, destacando las zonas en donde la comunicación interdisciplinal puede ser fluida (zonas de dependencia), donde puede ser conflictiva (zonas de conflicto) y donde la comunicación es inexistente (zonas autónomas), así para el autor

En la aplicación de las normas legales y reglamentarias del impuesto a las Ganancias y otros impuestos en Colombia, su vinculación con las normas y aplicaciones de los principios de la contabilidad, se puede apreciar la existencia de zonas de dependencia, de autonomía de ambas disciplinas y de conflicto, donde por falta de previsiones taxativamente establecidas en la norma tributaria, la materia contable puede tener algún grado de influencia en la interferencia (p. 1)

En cuanto a las zonas de dependencia que se visualizan por la aplicación de la norma tributaria colombiana este autor destaca el período fiscal, la anualidad del balance comercial y fiscal, el sistema de determinación del resultado impositivo ya que las empresas parten del balance comercial y vía ajustes se determinara la obligación tributaria, la aplicación en general del criterio de lo devengado en la imputación de ingresos y deducción de gastos y la valuación de inventarios. Para las zonas de independencia, este autor describe el desuso del concepto de devengado para las ventas a plazo y para los bienes de construcción de obra pública, la depreciación por trimestres y en función de una vida útil determinada en cincuenta (50) años, el sistema de depreciación según el método lineal, costo de las mercaderías vendidas, entre otros.

La existencia de zonas de independencia (autónomas) se debe a la aplicación de una norma tributaria, por lo que ante dichas exigencias muchos contribuyentes en Colombia (al igual que en Venezuela) han optado por dos opciones, o bien por llevar una doble contabilidad a efectos de cuantificar la renta gravable, o a que se tributarice la contabilidad por el peso del discurso tributario por sobre el discurso contable, ergo, a que sus registros contables se hagan con base en lo que la ley puede reconocer como Ingreso, Gasto, Costo, Pasivo o Activo. Se debe recordar que en algunos países las normas contables aún son emitidas por una institución distinta al Congreso de la República (caso Venezuela) lo que le quita peso y rango jurídico a su aplicación.

En muchas jurisdicciones se intenta resolver este conflicto a través de su régimen normativo. Para el caso del Impuesto sobre Rentas (ISR) "se remite al resultado contable como punto de partida normativo, regulando tan solo aquellas partidas que, a juicio del legislador, deban tener un tratamiento diferente para efectos del ISR empresarial" (Mejías et al 2015, p. 6) Tal 
es el caso de países como Alemania, Francia, Hong Kong, España e Italia, en consecuencia, las diferencias temporales que dan origen a Bases Financieras y a Bases Fiscales diferentes tienden a desaparecer, puesto que los hechos y transacciones deben reconocerse y registrarse y valorarse en concordancia con las normas contables y serán instrumentales a los fines de la Tributación.

Así, independientemente del tratamiento tributario o del grado en que la Tributación acuda al conocimiento contable para la determinación de las obligaciones (zonas de dependencia) la Contabilidad Financiera siempre será una herramienta para la Tributación.

Como se ha observado existen varias formas relacionales entre la Contabilidad Financiera y la Tributación. Los fenómenos que estas disciplinan abordan tienen una dinámica que le es propia y que van mutando a medida que dichos fenómenos se conectan con otros, es decir, estas disciplinas están condicionadas por el hecho de que los fenómenos que abordan están interconectados entre sí, dando origen a lo que Tamayo (2010) denomina como "estructuras de fenómenos" (p. 15) los cuales son más amplios y complejos que sus componentes y, a su vez, estas estructuras se interconectan nuevamente, presentando como resultado un tejido conceptual más complejo.

Esta interdisciplinariedad sugiere que la Tributación se ve en la necesidad de ampliar su tejido conceptual, su sistema de creencias, sus métodos de reconocimiento, necesitando en mayor o menor grado de los métodos, conceptos y principios contables para representar en un lenguaje tributario las obligaciones tributarias materiales que surjan de la relación jurídicotributaria entre las entidades comerciales y el Estado.

La Tributación también necesita de la Contabilidad Financiera para ser garantía metodológica y científica en cuanto a la preparación de información financiera con incidencia fiscal, a menos que, una ley o criterio jurisprudencial obvie el uso de los principios contables para el cálculo de las obligaciones tributarias y establezca criterios propios para el reconocimiento y medición de los hechos económicos que serán considerados como hechos imponibles. No obstante, como se observó, incluso para la asignación monetaria de la materia gravable, se debe acudir a la Contabilidad.

En contraste, la Contabilidad Financiera necesita en menor grado de la Tributación para el cumplimiento de sus fines ya que los hechos económicos que reconoce y mide la Contabilidad y que pueden estar sujetos a transformarse en obligaciones tributarias (Pasivos Tributarios que deben ser reconocidos en el Balance) representan, según Bastidas (2016), "un porcentaje relativamente bajo del universo de operaciones de las entidades lucrativas" (p. 5).

No obstante, eexisten tensiones en la interacción de ambos conocimientos que se visualizan en sus respectivos discursos y que probablemente son producidas por la divergencia existente en el sistema de creencias que subyace en cada disciplina. Nobes y Parker (1985) señalan que esas creencias están presentes en las siguientes categorías que hacen inestable al sistema contable y a su interacción con otros subsistemas: a) características del sistema legal; b) forma de financiación de las empresas que determina el tipo de usuario; c) conformación e influencia del sistema impositivo c) tamaño y fuerza de la profesión contable; d) nivel de inflación; e) enfoque de la teoría contable en cuanto a los objetivos asignados al sistema; f) acontecimientos de carácter económico o político que condicionan la respuesta de las normas contables.

Sin embargo, a continuación, se comentará la primera parte de una serie de reflexiones sobre algunas de las tensiones que a juicio del investigador pudieran tener su origen en lo disímil de cada sistema de creencias. Abordaremos la razonabilidad financiera como 
tensión disciplinal en la relación práctica entre Contabilidad Financiera y Tributación, pero antes, debo mencionar una diferencia epistemológica que me obliga a separar a la Contabilidad de la Contabilidad Financiera.

\section{Contabilidad Financiera Vs Contabilidad}

Hacer contabilidad no es igual a producir información financiera. La función de la contabilidad es registrar y presentar realidades económicas, es decir, intenta narrar la interacción económica de las empresas de la forma más fidedigna posible. No obstante, el proceso de globalización contable planteó la fusión de las finanzas y la contabilidad lo que diera origen a la Contabilidad Financiera. Hoy, las finanzas más que la economía, tienen una incidencia mayor en la Contabilidad Financiera lo que "produce que la contabilidad luche por definirse a sí misma en relación con los acontecimientos del pasado y la anticipación de eventos futuros que todavía no han ocurrido, o tal vez no ocurran" (Pacheco 2017)

La relación interdisciplinaria de la Contabilidad históricamente se produjo con la Economía y no con las Finanzas, aunque hubo una relación de cercanía, como previamente cité. Esta relación tan estrecha permitió que los Estados Financieros estuvieran dedicados casi exclusivamente a datos objetivos dado que a esta le interesaban sólo los hechos económicos y sus precios de intercambio. Sin embargo, visto el carácter predictivo (ex ante) de la Contabilidad Financiera, se hace evidente que los elementos de objetividad deben ser sacrificados.

En este sentido, es factible afirmar que si el registro histórico está ausente la contabilidad no realizará su función comunicativa (relatar - discurso) y no proporcionará una base de datos validada a los fines de la toma de decisiones de cara el futuro. Y aunque los informes financieros puedan definirse como reportes consecuencias de decisiones, compromisos y eventos que ya han ocurrido, es un acto meramente declarativo ya que el modelo contable internacional se basa en las expectativas y esto, en términos prácticos, se puede observar en la medición posterior de las partidas, el cual se basa en los posibles comportamientos de los sucesos que inciden en las valoraciones en el futuro de la entidad que reporta. Para este modelo el referente histórico es de poca utilidad.

La realidad es que la fusión de Contabilidad y Finanzas también ha generado una variedad de propuestas (algunas controvertidas) de medición que, dentro de los informes financieros, presentan igualmente cifras históricas basadas en el costo que son consecuencia de los hechos, y valores predictivos basados en flujos que son asociados con expectativas. Explica Hoogervorst (2012) que “(...) la multitud de técnicas de medición indica que los emisores de normas contables a menudo tienen dificultades para encontrar una respuesta clara a la pregunta de cómo un activo o pasivo debería valorarse" (p. 2) en función de las expectativas. En total, aclara el autor, los estándares internacionales emplean más de veinte (20) variantes basadas en el costo histórico o en valores predictivos.

Esta variedad en las formas de medición ocurre fundamentalmente por dos razones. La primera, la falta de una teoría generalmente aceptada de medición en contabilidad lo que hace que no se tengan referentes teóricos que sirvan de base para construir metodologías de medición y, la segunda, porque para las finanzas "el devengo" tiene muy poco valor. A las finanzas no le interesa lo que se haya vendido o comprado en términos fácticos, solo el resultado neto (el flujo neto de recursos futuro) Cabe aclarar que el "recurso" no es un concepto de la contabilidad, es un concepto eminentemente financiero.

Pareciera con esta fusión de la Contabilidad con las Finanzas que el objeto de estudio ya no son los Estados Financieros tal como tradicionalmente se conocían sino únicamente la información financiera, lo que obliga a que la Tributación tenga que desarrollar métodos para lidiar con nuevos conceptos, nuevos sintagmas, nuevos paradigmas e incluso con más imprecisiones y subjetividades que certezas. También pudiera obligar a que la Contabilidad Financiera desarrolle formas de conciliar tensiones desarrolladas hasta ahora, cuestión que parece difícil de materializar por los 
intereses y creencias de los productores del discurso.

Otra cosa para resaltar es la confusión por alevosía que el ente productor de normas hace al pretender que las entidades económicas en el mundo lleven Contabilidad Financiera como si fuera Contabilidad. Emular estas dos disciplinas equivaldría a pedirle a un abogado que aplique los principios del derecho mercantil al a las situaciones inherentes al derecho tributario. La Contabilidad Financiera es una contabilidad especializada, tal como lo es la bancaria, la de costos, la agropecuaria, la hotelera, la de seguros entre otras. Este error epistemológico será difícil de ser corregido dada las estructuras de poder en las que se mueven los entes productores de normas contables.

En conclusión, si la interacción entre ambas disciplinas bajo el esquema actual se mantiene, no sería tan irracional avizorar el desarrollo de una nueva arista de la Tributación para garantizar su efectiva comunicación con la Contabilidad Financiera que se denomine la "Tributación Prospectiva", pues se evidencia en la literatura especializada que el discurso tributario está poco dispuesto a ceder en su comunicación con otras áreas afines por el carácter imperativo, regulador y sancionador sobre el que está construido y por el afán recaudatorio de esta disciplina. Lo que sí es probable es que las tensiones disciplinales tenderán a profundizarse.

También es probable vislumbrar que la homogeneidad del discurso tributario se diluirá entre la multiplicidad de intereses que tienen los gobiernos de cada jurisdicción y entre los principios y metodologías que provienen del Derecho. Por otro lado, el discurso contable pareciera con más influencia global que el discurso tributario ya que proviene de estructuras de poder que tienen un peso muy importante en la generación de la riqueza mundial, lo que permitiría asegurar que lamentablemente en el mundo se seguirá obligando a las entidades a llevar Contabilidad Financiera en lugar de Contabilidad, aunque la mayoría de empresas en el mundo no participen en mercado de valores. Esta tensión es política.

\subsection{La razonabilidad de la información y la Tributación}

Una vez hecho esta separación ya es prudente mencionar el centro epistémico de esta reflexión, la razonabilidad de la información y su relación con la tributación. La razonabilidad financiera es un producto de la aplicación del modelo de regulación contable internacional. Para este modelo, la información financiera será razonable, si y solo si, se aplican todas las reglas que este modelo tipifique, lo que quiere decir que un reconocimiento o medición distinto a los que están en ellas contenidos hará que se pierda esta cualidad. No obstante, la Tributación no contempla la razonabilidad de la información tal y como lo concibe la Contabilidad Financiera, pues ella prima la certeza por sobre la incertidumbre. A la Tributación le interesa la realidad material no la realidad proyectada.

Según la Norma Internacional de Contabilidad 1 (NIC 1) en su párrafo 15, los Estados Financieros deben reflejar razonablemente, la situación, el desempeño financiero y los flujos de efectivo de la entidad. Esta presentación razonable exige proporcionar la imagen fiel de los efectos de las transacciones, así como de otros eventos y condiciones, de acuerdo con las definiciones y los criterios de reconocimiento de Activos, Pasivos, Ingresos y Gastos fijados en el Marco Conceptual $\mathrm{y}$ todos los requisitos de las normas internacionales.

Al asumir que la aplicación de las NIIF, acompañada de información adicional cuando sea necesario, dará lugar a Estados Financieros que proporcionen una presentación razonable es una falacia de petición de principio $^{1}$ por parte del ente emisor de normas, pues la razonabilidad de los Estados Financieros no solo debe ser "consecuencia

1. Sobre la degradación de la representación fiel en el modelo de regulación contable internacional y su vinculación con la relevancia más que con la fiabilidad de la información ver Wüstemann, Sonja. Does faithful representation mean reliability? Working Paper, Goethe University Frankfurt am Main Version 4.0, as of 24 April, 2011. 
de un juicio de conformidad con algún marco técnico de referencia" (Romero- Muci 2011 p. 48), sino debe ser su grado de correspondencia con los hechos que se dan en la empírea económica.

También, cabe destacar otros requerimientos de la NIC 1. Esta norma no contempla los flujos de efectivo futuros de la entidad (solo de flujos de efectivo), en cambio, el Marco Conceptual (2018) contempla reflejar la entrada o salida de los flujos de efectivo en el futuro como requisito para representar fielmente las partidas contables expresadas en los Estados Financieros; he aquí otro problema representacional que tiene el modelo internacional al mezclar valores objetivos y subjetivos en el cuerpo de los Estados Financieros.

A pesar de que la representación de la información fiel (que da origen según el modelo internacional a la información razonable) tiene según el Marco Conceptual (2018) tres características, es decir, que sea completa, neutral y libre de errores, en opinión del investigador es solo una declaración retórica en el discurso normativo contable, incluso casi manipuladora por parte del IASB.

Por ejemplo, la neutralidad es un concepto que no es multidimensional y no tiene implícitos los conceptos de incertidumbre o de predicción de eventos futuros. Aunque no existe consenso en su delimitación conceptual, puede enmarcase en una dicotomía fáctica, es decir, no se puede ser parcialmente neutro, o se es neutro o no se es. En términos temporales, la neutralidad es aplicable ante situaciones que ocurrieron sin entrar a analizar o justificar las razones que les dieron origen. La neutralidad no es aplicable para situaciones que están por ocurrir.

En este sentido, la contabilidad al relatar los hechos económicos trata de no tomar partido por los sucesos de la empírea económica que ocurrieron, simplemente los comunica para que la gerencia y otros usuarios puedan tomar decisiones. No se les puede pedir a los practicantes de la disciplina que sus representaciones sean neutrales en un contexto de estimaciones y de juicios de valor como en efecto pasa en la Contabilidad Financiera. El acto de predecir no es un acto neutro, es una acción de juicio subjetivo entre varias opciones en las que se obvia la realidad tal y como es y se propone tal y como pudiera ser a juicio del practicante. Este es un acto influenciado (sesgado) por el mercado, creencias sobre el sistema financiero, expectativas, experiencia profesional, modelos mentales, ideologías, entre otras cosas.

Por otro lado, desde el punto de vista contable la razonabilidad pudiera ser definida como la cualidad que tienen los Estados Financieros de ser razonables. Esto no es circularidad en el lenguaje, simplemente existe la razonabilidad cuando los Estados Financieros son razonables, de lo contrario, no existirá. Es como afirmar que existe la solidaridad en una persona producto de su comportamiento solidario; sin comportamiento solidario no hay presencia de la solidaridad en la persona. No obstante, como se mencionó, para el IASB la razonabilidad es otra cosa.

En opinión del investigador, para que la elaboración y presentación sea razonable debe estar conforme a la razón, y los Estados Financieros estarán conforme a la razón cuando las transacciones y los acontecimientos se reconozcan y contabilicen en función del correlato fáctico que les dio origen, es decir, debe existir una coherencia entre el correlato fáctico, su reconocimiento, su medición y la posterior aseveración hecha de ésos correlatos en los Estados Financieros.

Por lo tanto, si los encargados del diseño del modelo de regulación contable internacional quieren aplicar este concepto, deben privilegiar la realidad existencial por sobre la estimación de la misma. No es que se obvie la cuantificación como forma de comunicar información económica asociada al intercambio de bienes y servicios, es que se privilegie los precios de transacción por sobre los valores de predicción en el proceso de relatar los hechos y transacciones de una entidad.

Estimar un beneficio económico no es reconocer la realidad, es predecirla, y esto no puede ser considerado como imagen o representación fiel. En tal caso, el enunciado más apropiado sería 
“estimación fiel". Incluso el sintagma "estimación fiel" es un tanto ambicioso en cuanto a su extensión, así que, como se citó, pudiera dársele cabida al sintagma "estimación probable" y con una aplicabilidad única en el modelo de regulación contable internacional para aquellas empresas que coticen en el mercado de valores. El modelo de regulación contable internacional debe ser aplicable solo a este tipo de empresas.

Como se dijo, es creencia del investigador que desde el punto de vista contable la razonabilidad es la cualidad que tienen los Estados Financieros de ser razonables y, para que éstos sean razonables, tienen que estar preparados y presentados conforme a la razón. Ahora, ¿Cuándo el reconocimiento de una transacción o hecho económico está conforme a la razón, para que posteriormente tengan la cualidad de ser razonables en el cuerpo de los Estados Financieros y por ende, se configure la razonabilidad en los EEFF en su conjunto? A juicio del investigador existen muchas vías, pero, una práctica es cuando se cumplan de forma concurrente con los siguientes principios que vienen de la lógica de Aristóteles y luego perfeccionados por Leibeniz:

\section{a) El principio de identidad}

El cual evidencia que un concepto es ese mismo concepto (A es A). Por ejemplo, el concepto de "Ingreso" contiene el incremento de beneficios económicos según el modelo internacional, no los posibles incrementos. Ningun otro concepto en Contabilidad Financiera puede representar hipotética y simbólicamente los hechos que involucren el aumento de beneficios económicos, solo el concepto de "Ingreso" tiene la identidad para hacerlo. Ingreso es Ingreso, no es Egreso ni Activo, ni es Pasivo. El problema bajo el modelo actual surge cuando se incorporan valores probables para medir el "Ingreso" y no aquellos que dicte la empírea, la identidad que surge a partir de la única consideración de su concepto se ve afectada, no hay una realidad monetaria ocurrida, sino por ocurrir. Aunque el investigador está consciente de la teleología del modelo internacional hay serias consecuencias epistemológicas en la interacción disciplinal con la Tributación.

\section{b) El principio de no contradicción}

Esta evidencia que un mismo concepto no puede ser y no ser a la vez (A no es negación de A) Un mismo hecho no puedo ser reconocido de formas distintas en contabilidad y así ser reflejado en los Estados Financieros. Ejemplo, según el modelo internacional un arrendamiento financiero es un Activo controlado por la entidad que reporta $y$ debe ser reconocido como Activo, no puede ser reconocido como gasto, pues al hacerlo, sería la negación del concepto de activo que considera el constructo del "control". Este principio no se ve reflejado en el modelo internacional, por ejemplo, para el caso de los Activos Intangibles desarrollados por la propia empresa en el que a pesar de cumplir con todas las definiciones de Activo, el modelo internacional decide denotarlo como un Gasto.

\section{c) El principio del tercero excluido}

Sugiere que entre el ser o no ser de un concepto, no cabe situación intermedia (A es A, o no lo es, no hay parcialidades de A) Por ejemplo, un Pasivo o es contingente o es Pasivo, o se revela o en su defecto se cuantifica y se presenta, en vista de que A no es $\mathrm{B}, \mathrm{A}$ es A y no hay intervalos de reconocimiento de A. No existen Pasivos intermedios que hagan que el Pasivo pueda ser o no al mismo tiempo. O es Pasivo contingente o es Pasivo. Por ejemplo, para el caso de unos posibles litigios por demandas ambientales, mientras sale la decisión de la demanda se tiene un Pasivo Contingente, al salir el fallo se tendrá un Pasivo (si involucra algún tipo de desembolso)

\section{d) El principio de la razón suficiente,}

Evidencia que toda idea verdadera debe tener suficiente fundamentación para que soporte los procesos de falsación. Así, por ejemplo, la representación fiel que la Contabilidad Financiera pretende argüir en su modelo, debería tener el soporte empírico y normativo suficiente para coadyuvar en la representación de la realidad económica de las empresas que hagan los practicantes de esta disciplina tal y como se dieron, y no representar un conjunto de percepciones como en realidad pasa. Este concepto del IASB es válido en un contexto 
de mercado de valores, no de empresas que no estén en ese sistema y, lamentablemente, el modelo pretende ser universal para todas las empresas. Es imposible fundamentar suficientemente una tesis falsa, y por más que el IASB asegure y fundamente que hace representación fiel, no podrá hacerlo, pues su tesis de representación fiel es en algunos casos impracticable.

Sin ánimos de pretender que estos principios se conviertan en aproximaciones teóricas para el desarrollo de una teoría sobre fiabilidad, cuya ausencia demanda elocuentemente Wuestman (2011), si se pretende a través de ellos comprobar que el modelo de regulación contable internacional, al hacer estimaciones probables en la medición posterior de los hechos y transacciones que irán al cuerpo de los Estados Financieros, lo que causa es que ya no se representen fehacientemente los fenómenos de la empírea sino lo "relevante" a los efectos de los usuarios previstos. Por ejemplo, véase el caso de las "Provisiones", las cuales son Pasivos con cuantía o vencimiento inciertos.

Señala la Sección 27 (IASB PYME) que las entidades reconocerán la provisión como un Pasivo en el estado de situación financiera, y el importe de la provisión como un gasto. ${ }^{2}$ La medición posterior para estos casos pareciera también verse influida por el efecto predictivo del modelo, puesto que existe el deber de cargar contra la "provisión únicamente los desembolsos para los que fue originalmente reconocida y de ajustar las provisiones en cada fecha sobre la que se informa para reflejar la mejor estimación actual del importe requerido para cancelar la obligación en esa fecha" (párrafo 21.11 eiusdem)

Empero, esa provisión por obligación también pudo haberse calculado en un momento inicial bajo la estimación del valor presente de los importes que se espera sean requeridos para liquidarla en el futuro si el efecto del valor del dinero en el tiempo resultase significativo (párrafo 21.11 eiusdem) En este contexto los valores de la provisión no reflejarán hechos de la empírea, lo relevante, a los fines de los usuarios previstos, es hacer estimaciones de los posibles desembolsos sobre esta posible obligación.

En este sentido, si la Tributación utiliza enteramente a la Contabilidad Financiera de forma instrumental para fines de determinación, pudiera encontrarse con gastos producto de una obligación futura en el Estado de Resultados que no tienen un correlato fáctico, por lo que existiría incerteza sobre el monto a deducir y, tal vez, los productores del discurso tributario deberían poner en entre dicho esa valoración para el caso concreto del Impuesto a las Ganancias. Si bien parece normalizado este criterio en la corriente legalista lo que hace es desproveer a la Contabilidad de su rol probatorio ante terceros ya que hace aseveraciones que en efecto no existen, solo que pudieran existir. Lo que es obvio es la aparición de un Impuesto Diferido en el caso de que las Bases Fiscales y las Bases Financieras para este tipo de obligación sean diferentes.

En este orden, cabe citar las palabras de Pacheco (2013) en el que afirma que el IASB es "realista ingenuo" cuando pretende que el objetivo de la utilidad de la información sea satisfecho a través de la representación fiel. A la representación fiel no debe interesarle si la información es útil, solo debe reflejar en un lenguaje contable y con un alto grado de correspondencia con la realidad, todos los fenómenos y transacciones de la empírea económica que rodea a una determinada entidad; la Contabilidad debería comportarse como un espejo factual que refleje fielmente lo que pasó en la realidad económica. Luego, los usuarios tomarían las decisiones que consideren pertinentes con base en esa información que debe comunicarles la Contabilidad.

En el proceso de identificación de los fenómenos económicos no debe existir ningún filtro, como en efecto lo plantea el párrafo 2.21 del Marco Conceptual (2018), lo cual va en contra de lo que

2. A menos que otra sección de esta NIIF requiera que el costo se reconozca como parte del costo de un activo tal como inventarios o propiedades, planta y equipo. Párrafo 27.5 IASB PYME.

Bastidas Canelones, Jorge

Breve reflexión sobre la razonabilidad de la Información Financiera y la Tributación 
tradicionalmente se conoce en la doctrina contable como representación fiel. Representar fielmente la sustancia de los fenómenos que se pretende representar es impracticable si se hacen estimaciones valorativas de esos fenómenos y se pasan por el tamiz de la relevancia y la utilidad informativa para un determinado grupo de usuarios.

Cabe preguntarse, ¿Cómo una información relevante sobre un fenómeno que tenga una estimación altamente incierta proporcionaría una representación suficientemente fiel de cómo ocurrió ese fenómeno? Es imposible, de allí el calificativo de realista ingenuo. El IASB concibe como válidas informaciones útiles aún y cuando tenga estimaciones altamente inciertas; esa no es información representada fielmente y la Tributación debe lidiar con estas situaciones en los procesos de determinación de las obligaciones tributarias.

\section{Conclusiones}

En resumen, en opinión del investigador, la aplicación del modelo de regulación contable internacional en ocasiones produce distorsiones en la representación de la realidad económica que circundó a la entidad que reporta, por lo que podría afirmarse que este modelo pudiera ser una interfaz inadecuada para representar fielmente las transacciones o hechos económicos que se dan en la realidad, en consecuencia, tampoco el modelo sería una base metodológica o instrumental confiable para la determinación de obligaciones tributarias. Sin embargo, en la praxis disciplinal ambos conocimientos se ven obligados a interactuar de forma constante, dada la pretensión del IASB de hacer Contabilidad Financiera en lugar de Contabilidad, y a la juridificación que se ha visto en el mundo sobre las normas contables emitidas por este organismo.

En definitiva, los Estados Financieros originados a partir de la aplicación de este modelo no deben ser confundidos con un juicio de certeza absoluta sobre los hechos económicos dada las estimaciones presentes en la medición de la información, lo que le da un carácter subjetivo a la información financiera, la llena de incertezas en la medición de los hechos económicos y origina incerteza en la medición de las obligaciones tributarias cuando se use a la Contabilidad Financiera de forma instrumental con fines de determinación impositiva. La información financiera es valorativa y descriptiva de los hechos relevantes que allí se sintetizan, pero no es un juicio de certeza como bien sostiene Romero-Muci (2011 p. 82)

Somos del criterio que la información financiera construida aplicando el modelo internacional no es razonable en los términos de lo que la palabra razonable significa para el lenguaje común, ergo, información reconocida y procesada fundamentada en la lógica y en la razón. Resulta que el "lenguaje ordinario y el contable no son independientes, sino que están estrechamente relacionados ya que el segundo se desarrolla a partir del primero" (López 1998 , p. 1) por lo que el lenguaje ordinario no debe ser obviado en el estudio interdisciplinario, ya que es la base comunicativa de la interacción disciplinaria y a partir de allí debería construirse una semántica especializada de uso común, pero siempre considerando la semántica y semiótica del lenguaje ordinario.

Información razonable no es información ajustada a la razón bajo el enfoque del modelo de regulación internacional, es información útil para ciertos usuarios y con ciertos propósitos. $\mathrm{Si}$ el modelo quiere ser coherente se propone que la información razonable debería denotarse como "información probable" pues tiene pocos referentes empíricos y depende de la probabilidad de que las estimaciones sean acertadas. Al menos el uso de este sintagma sería más congruente con la teleología del modelo.

Bajo este modelo, la información financiera solo es construida para ser útil a los fines de los que toman decisiones en el mercado de valores, y aún si fuera razonable (acorde con la razón) aplicando las reglas del modelo, su uso para fines fiscales debiera tener la condición de verificable, es decir, que "tenga existencia" (Li 1963 p. 104) y que no se abuse de las estimaciones; o al menos que estos juicios y estimaciones no sean el punto neurálgico de la representación. 
La razonabilidad, tal y como lo entiende el modelo internacional, admite en mayor grado estimaciones (no verificables con un hecho de la empírea) en lugar de exactitudes; y la Tributación tiene que lidiar con esta forma de representar la realidad en los procesos de determinación impositiva. Dicho de otro modo, la Tributación recibe de la Contabilidad Financiera hechos relevantes y no hechos o transacciones fielmente representados. Esta reflexión permite al autor pensar que esta tensión es ontológica, en próximos artículos se estará comentado sobre otras tensiones en la relación interdisciplinar entre Contabilidad Financiera y Tributación.

\section{Referencias bibliográficas}

American Accounting Asociación (1966). A Statement of Basic Accounting Theory (ASOBAT). American Accounting Association Publications. Estados Unidos de América.

Arreghini, H. (2012). Contabilidad: Sus fundamentos conceptuales. Resumen de tesis doctoral. Argentina: Universidad de Buenos Aires. Disponible en http://ojs.uba.ar

Arthur Andersen \& Co. (1972). Objetivos de los Estados Financieros para las Empresas de Negocios. Arthur Andersen \& Co.

Arthur Andersen \& Co. (1974). Normas de Contabilidad para Empresas de Negocios en todo el mundo. Arthur Andersen \& Co.

Baudrillard, J. (1978). Cultura y Simulacros. Barcelona, España: Editorial Kairos.

Baudrillard, J. (1998). Simulacros y simulaciones. Estados Unidos: Stanford University Press.

Belkaoui, A. (1993). Accounting Theory. Hartcourt Brace, Jovanovich College Publishers, Gran Bretaña.

Berger, P. y Luckmann, T. (2001). La construcción social de la realidad. Argentina: Amorrotur Editores (décimo séptima impresión).
Biondi, Y., Glover, J., Jamal, K., Ohlson, J., Penman, S., Sunder, S. y Tsujiyama, E. (2012). Some Conceptual Tensions in Financial Reporting. Estados Unidos de América. Accounting Horinzons. Volumen 26. Número 1.pp. 125133. Disponible en http://aaajournals.org

Boter, F. (1959). Las doctrinas contables. Editorial Juventud, S.A., Barcelona, España. Burton, J. (1976). Conflicts and compromises in financial reporting. Disponible en www.sechistorical.org

Cañibano, L., Tua, J., López, J. (1985). Naturaleza y filosofía de los principios contables. Revista Española de Financiación y Contabilidad. Volumen XV, número 47, pp. 293-355.

Cañibano, L. (1975). Teoría de la Contabilidad. Ed. ICE, Madrid, España.

Day, R. (2000). UK accounting regulation: an historical perspective. School of Finance \& Law Working Paper Series. Número 20. Bournemouth University.

Demkis, J., Fellingham, J., Ijiri, Y. y Sunder, S. (2002). Some thoughts on intellectual foundations of accounting. Conferencia de la Universidad Carnegie Mellon. Publicado en American Acoounting Association: Acounting Horizons. Volumen 16, número 2. pp. 157168. Disponible en www.citeseerx.ist.psu.edu

Diccionario de la Real Academia de la Lengua Española. Versión electrónica. Disponible en http://www.rae.es/recursos/diccionarios/drae

Durán y Mejías (2013). La interdisciplina tributaria. Lima, Perú.

Dussel, E. (2000). Sobre el Sujeto y la Intersubjetividad; El Agente Histórico como Actor en los Movimientos Sociales. Disponible en www.Pasos841.htm (búsqueda realizada en 2018).

FASB e IASB (2006). A Roadmap for Convergence between IFRSs and US GAAP-2006-2008. 
Memorandum of Undrestanding between the FASB and the IASB. Statement, Conceptual Framework for Financial Reporting. Norwalk, Estados Unidos de América. Disponible en www.ifrs.org

Ferullo y otros (2000) La contabilidad como base de la determinación tributaria. Quintas Jornadas "Investigaciones en la Facultad" de Ciencias Económicas y Estadística, Noviembre de 2000. Universidad del Rosario.

Financial Accounting Standards Board (FASB) and the International Accounting Standards Board (IASB) (2018). Preliminary Views, Conceptual Framework for Financial Reporting: Objective of Financial Reporting and Qualitative Characteristics of Decision-Useful Financial Reporting Information. Norwalk, Estados Unidos de América. Disponible en www.ifrs. org.

Hevia, B (2010) Arrendamiento Financiero e Impuesto sobre la Renta. Caracas Venezuela.

Ley de Impuesto sobre Sucesiones, Donaciones y demás Ramos Conexos. Venezuela. (1982).

Norma Internacional de Contabilidad 12. Impuesto a las Ganacias. IASB (2018).

Pacheco, J (2013) Los conflictos en Contabilidad a luz del modelo de regulación contable internacional. Tesis doctoral. Mérida, Venezuela.

Romero Mucci, H (2009). El Derecho y el Revés de la Contabilidad. Caracas, Venezuela.

Searle J. (1997) La construcción social de la realidad. México. Paídos.

Sentencia Corte Suprema de Argentina caso "Compañía Tucumana de Refrescos S.A" (s.a)

Tucker, B. y Lowe, A. (2014). Practitioners are from Mars; academics are from Venus?: An investigation of the research-practice gap in management accounting. Accounting, Auditing \& Accountability Journal, 27(3), 394-425 